\title{
CAMBio CLIMÁTICO EN LA REgión Noreste del URUguay: CLIVAJES EN LAS PERCEPCIONES DE LOS ACTORES TERRITORIALES
}

Amalia Margarita Stuhldreher ${ }^{*}$

\section{Resumen}

Después de diez años de la creación del Sistema Nacional de Respuesta al Cambio Climático (SNRCC) del Uruguay, este trabajo analiza la percepción de los actores territoriales acerca de los impactos del cambio climático en la región Noreste del país (departamentos de Rivera, Tacuarembó y Cerro Largo), así como sus visiones acerca de las políticas y los mecanismos de la gobernanza climática del Uruguay, focalizando aspectos como la articulación y la participación. Se apunta aquí a una reflexión que genere insumos relevantes para el diseńo específico de políticas exitosas de mitigación y adaptación a dicho fenómeno en el territorio analizado.
Palabras clave: desarrollo sostenible; cambio climático; gobernanza; región Noreste de Uruguay.

\section{CLIMATE CHANGE IN THE NORTHEAST RE- GION OF URUGUAY: PERCEPTION CLEAVAGES AMONG LOCAL ACTORS}

\begin{abstract}
Ten years after the creation of the National System of Response to Climate Change (sNRCC for its acronym in Spanish) in Uruguay, this paper examines the perceptions among local actors about the impacts of climate change on the Northeast region of the country (De-
\end{abstract}

$\mathrm{PhD}$ en Ciencia Política. Profesora del Instituto de Desarrollo Sostenible, Innovación e Inclusión Social (IDIIS), sede Tacuarembó, Universidad de la República (UDELAR) (Uruguay). [astuhldreher@hotmail.com], [https://orcid.org/0000-0002-0918-3099].

Recibido: 5 de agosto de 2019 / Modificado: 25 de septiembre de 2019 / Aceptado: 6 de diciembre de 2019 Para citar este artículo

Stuhldreher, A. M. (2020). Cambio climático en la región Noreste del Uruguay: clivajes en las percepciones de los actores territoriales. OPERA, 27, 181-191.

DoI: https://doi.org/10.18601/16578651.n27.09 
partments of Rivera, Tacuarembó and Cerro Largo). It also analyzes their visions about policies and mechanisms of climate governance defined at the national level, focusing some aspects such as articulation and participation. This way the paper aims to generate relevant inputs for the discussion about the design of policies for mitigation and adaptation to this phenomenon in the specific territory considered here.

Key words: Sustainable development; climate change; governance; Northeast region of Uruguay.

\section{INTRODUCCIÓN}

Desde una perspectiva de gobernanza territorial multinivel, y después de diez años de la creación del Sistema Nacional de Respuesta al Cambio Climático (ssnrcc) del Uruguay, este trabajo analiza la percepción de los actores territoriales acerca de los impactos del cambio climático en la región Noreste del país (departamentos de Rivera, Tacuarembó y Cerro Largo), así como los posibles clivajes en sus visiones acerca de las políticas y los mecanismos de la gobernanza climática del Uruguay, considerando aspectos relativos a articulación y participación. Se apunta aquí a una reflexión que pueda proporcionar insumos relevantes para el diseño específico de políticas exitosas de mitigación y adaptación a dicho fenómeno en el territorio considerado. En ese sentido, puede señalarse que estas acciones (y su anclaje institucional) debieran tener en cuenta los clivajes en las percepciones de diversos actores sobre este fenómeno en cuanto a su alcance, implicancias y posibles estrategias para este territorio en particular.

En términos de impacto, siguiendo los datos de la edición 2019 del Índice de Riesgo Climático Global (IRC) ${ }^{1}$ de Germanwatch, es posible señalar que países de América Latina y el Caribe, como Puerto Rico y Honduras, figuran entre aquellos que de 1998 a 2017 fueron más afectados por el cambio climático. De los países más afectados en el índice a largo plazo, la mayoría tiene un alto ranking debido a catástrofes excepcionales. A esto se suma recientemente otra categoría de países que se ven impactados de manera constante por catástrofes, como Haití. Por otra parte, los resultados ICR 2019 (que tiene como base datos de 2017) indican que Puerto Rico, Sri Lanka y Dominica fueron los países más afectados en 2017, seguidos por Nepal, Perú y Vietnam (Germanwatch, 2019). En términos de percepciones, de acuerdo con datos de INTAL/ Latinobarómetro 2017, se constata que Uruguay es el país de América Latina donde más personas consideran que el problema del cambio climático existe $(87 \%)$. En contraste con esto, en el extremo opuesto, en Ecuador solo lo percibe así el $42 \%$. Por otra parte, al comparar las respuestas con el Índice de Vulnerabilidad

1 El Índice de Riesgo Climático Global (Irc) de Germanwatch es un análisis basado en uno de los conjuntos de datos más fiables disponibles sobre los impactos de los eventos climáticos extremos y los datos socioeconómicos asociados a ellos, tomando como indicadores el número de muertos por las catástrofes climáticas, cantidad de muertos por 100.000 habitantes, pérdida en millones de dólares (PPA), pérdidas por unidad de producto interno bruto (PIB) (en \%), así como Índice de Desarrollo Humano (IDH) (Germanwatch, 2019). 
al Cambio Climático², se constata una correlación negativa $(0,57)$. Esto indica que los países que estarían más expuestos al cambio climático son aquellos que menos perciben el problema: República Dominicana (46\%), Guatemala (50\%), Nicaragua (50\%), El Salvador $(51 \%)$. En forma inversa, los menos expuestos son aquellos que más lo perciben: Brasil (73\%), Colombia (74\%), Argentina (77\%) y Uruguay (87\%). Respecto al impacto del cambio climático, los latinoamericanos ubican en primer lugar las consecuencias sobre la salud humana (14\%), en segundo lugar, la flora y la fauna $(12 \%) \mathrm{y}$, en tercer lugar, la vida cotidiana (11\%) (Basco, 2018).

Según Basco (2018) podría suponerse que en la medida en que el problema del cambio climático es visualizado, mayores serán los esfuerzos de los países para poner en marcha estrategias adaptativas o de mitigación. El diseño de políticas obligaría a revisar las capacidades institucionales para dar respuesta al problema, es decir, para servir de marco eficaz y eficiente para una gobernanza ambiental, mientras que la aplicación de esas políticas exigiría una

2 El Índice de Vulnerabilidad al Cambio Climático (IVCC) para América Latina y el Caribe analiza la vulnerabilidad de las poblaciones humanas a fenómenos extremos relacionados con el clima, así como los cambios en los principales parámetros del clima en los próximos treinta años. Combina el riesgo de exposición y los cambios en el clima y fenómenos extremos con la actual sensibilidad humana a esa exposición, así como la capacidad de un país para adaptarse o aprovechar los posibles cambios climáticos. Esta relación entre la profundidad de los cambios a los que está expuesto un país y la resiliencia existente en la población y el Gobierno de dicho país muestra un panorama general del grado de vulnerabilidad (CAF, 2014). participación activa de la ciudadanía. De esta manera, la conciencia social sobre el medio ambiente y el cambio climático devendría en un punto de partida crucial en la estrategia adaptativa de los países.

Al mismo tiempo, cabe esperar que las posibilidades de éxito de dichas políticas se verán decisivamente afectadas por las percepciones de los actores locales y su disposición al compromiso con las estrategias pergeñadas en el nivel nacional. Significativamente, en los Estados latinoamericanos se constatan dificultades en materia de coordinación e integración a la hora de diseñar estrategias para dar respuesta a los desafíos del cambio climático. En numerosos casos resulta evidente la existencia de una brecha entre los niveles nacional, local y regional, impregnados por lógicas de acción distintas como producto de la diversa evolución económico-productiva y sociocultural (Stuhldreher, 2016).

El presente artículo define, en primer lugar, un abordaje teórico-conceptual para encuadrar el análisis de los desafíos en materia de cambio climático desde el punto de vista de la gobernanza territorial multinivel en el Uruguay, con foco en la región Noreste. En segundo lugar, delinea aspectos centrales de la institucionalidad y los mecanismos definidos en el país en esta temática, que representan el marco de acción para los diferentes actores involucrados tanto a nivel nacional como lo$\mathrm{cal} /$ territorial. En tercer lugar, se explicita la estrategia metodológica. En cuarto lugar, se presentan los resultados del estudio de las percepciones de los actores de los departamentos frente a políticas y acciones que en Uruguay se definen principalmente desde el nivel nacional. 
Se identifican en ese contexto percepciones diferenciales de actores del ámbito gubernamental, así como de la sociedad civil entendida en un sentido amplio neoweberiano (como, por ejemplo, grandes y pequeńos productores y sus respectivas agremiaciones, academia, profesionales y técnicos, organizaciones sociales, organizaciones no gubernamentales [ONG], etc.). Finalmente, en las conclusiones se discuten los hallazgos del estudio y se esbozan algunas perspectivas a futuro.

\section{ABORDAJE TEÓRICO-CONCEPTUAL: LA PERSPECTIVA DE LA GOBERNANZA TERRITORIAL MULTINIVEL}

Considerando las implicancias del fenómeno del cambio climático, con múltiples riesgos (inundaciones, sequías, oleadas de calor, tornados, etc.) para un territorio con las características económico-productivas, ambientales y sociales propias de la región Noreste, se justifica un abordaje que focalice la percepción de actores territoriales involucrados en la problemática. Ya la Declaración de Río de 1992 subrayó expresamente la necesidad de reconocer el rol fundamental de las comunidades locales en el ordenamiento del medio ambiente y en la promoción del desarrollo sostenible debido a sus conocimientos y prácticas tradicionales. Tras el Acuerdo de París (2015) una serie de iniciativas tales como Gobiernos Locales para la Sustentabilidad (ICLEI, por sus siglas en inglés) o el Grupo de Liderazgo Climático (conocido como C40) han puesto de manifiesto a nivel internacional impulsos provenientes del nivel local/territorial. También, en el marco de la Agenda Pos-2015, los Objetivos de Desarrollo Sostenible (oDs), y específicamente el Objetivo 13 relativo al cambio climático, incluyen expresamente el nivel territorial.

El análisis de los desafíos del cambio climático debe contemplar, asimismo, los contextos regional y global, con sus implicancias en términos de gobernanza multinivel. Actualmente, América Latina se ve confrontada con la paradoja de una asimetría fundamental: pese a que la región ha tenido una contribución históricamente menor al cambio climático en comparación con otras regiones del mundo, por otra parte es particularmente vulnerable a sus efectos y estará involucrada necesariamente en la búsqueda de soluciones (CEPAL, 2014, p. 9). Aunque el cambio climático es priorizado por encima de muchos otros desafíos de las sociedades latinoamericanas (incluso en detrimento del crecimiento económico, según el $71 \%$ de los encuestados por el Latinobarómetro 2017), surge el desafío de la articulación de esfuerzos de actores públicos y privados en el diseño e implementación de políticas de mitigación y adaptación al fenómeno. Es evidente que la integración de América Latina a la economía global exige revisar aspectos de la gobernanza ambiental, en un contexto en el que los latinoamericanos identifican que quienes deben ponerse al frente de esta cuestión son tanto la totalidad de los actores sociales $(35 \%$ de las respuestas de la encuesta mencionada), así como las instituciones específicamente relevantes (gobiernos, organizaciones no gubernamentales, organismos internacionales, empresas, con un 32\% de dichas respuestas) (Basco, 2018). 
En el caso uruguayo puede decirse que existe una comprensión creciente de que la lucha contra el cambio climático debe involucrar a todos los actores y niveles de decisión (López, 2009, p. 3), lo que procuró ser contemplado en la institucionalidad definida desde la creación en 2009 del snrcc. Para un territorio como el de la región Noreste del país, rezagado en su desarrollo relativo (Laurnaga y Pastori, 2016), con fuerte impronta agropecuaria y donde el desempeño de los agroecosistemas depende directamente de los efectos positivos o negativos del clima, surge el desafío de conciliar las legítimas aspiraciones de progreso con los criterios de sustentabilidad social y ambiental. Esto puede implicar clivajes en la percepción de un fenómeno como el cambio climático, lo que a su vez genera desafíos en términos de gobernanza.

En términos teóricos y de evolución epistémica, originalmente el enfoque de gobernanza estuvo asociado a la economía institucional, cuyos antecedentes se remontan a las primeras décadas del siglo xx, con la escuela de la regulación, surgida en los años setenta, en un contexto de crisis donde se cuestionaban aquellas formas institucionales políticas y sociales que habían regulado la economía. En ese sentido, la categoría conceptual de la gobernanza se vinculó con el mercado, buscando mejorar los procesos de regulación e intervención pública y facilitando la toma de decisiones del resto de los actores sociales. La discusión del concepto, en el marco de la ciencia política y la administración, condujo al abordaje de esquemas relacionales impregnados por una lógica horizontal: se focalizó el desarrollo de una gestión de tenor más inclusivo, con un espectro diverso de agentes sociales asumiendo responsabilidades, en contraste con los modelos más tradicionales-burocráticos con primacía de la imposición. Al ser un concepto polisémico, fue incorporado por distintas corrientes teóricas, desde la nueva gerencia pública de la nueva derecha, el neoinstitucionalismo o el universalismo comunitario de la sociedad civil y las organizaciones no gubernamentales (Farinós, 2008, p. 12).

Recuperando la categoría de gobernanza, Renate Mayntz (2000) enfatizó la capacidad generada en los territorios para administrar los asuntos públicos a partir del compromiso conjunto y cooperativo de los actores institucionales y económicos de esos ámbitos. En contraste con la conceptualización de gobierno - vinculada a la presencia del poder del Estado articulado en un sistema de relaciones jerárquicas y burocráticas- la gobernanza implica un sistema de relaciones más amplio que abarca a otros actores no tradicionales del ámbito político, lo que conlleva un modelo alternativo e innovador de gestión de los asuntos públicos (Carmona, 2006; Farinós, 2008, p. 12). La interacción y la cooperación devendrían en la piedra angular de la vinculación de los actores y niveles de gobierno. Estas dinámicas novedosas crearían nuevos marcos institucionales, formales e informales, dentro de los distintos espacios territoriales. Esto determinaría un nuevo marco de ordenamiento legal, pero, sobre todo, permitiría la generación de redes y espacios de participación pública, donde junto a los elementos formales pesarían fuertemente los vínculos gestados a partir de la creación de confianza y el conocimiento de los participantes (Kooiman, 2003; Moncayo, 2002). 
Estudios de caso sobre políticas climáticas subrayan la importancia del tipo de relaciones que se establecen entre los actores, a la vez que señalan que la inclusión de un espectro de actores varios (expertos, grupos sociales, ciudadanos) resulta un pivote central para que la experticia y la aceptación necesarias sean incluidas en la toma de decisiones y el diseño de políticas legítimas, que luego puedan ser efectivamente implementadas (Kronsell, Khan y Hildingsson, 2019). En ese contexto, siguiendo a Farinós Dasi (2015), puede asumirse que la noción de gobernanza brinda una nueva perspectiva para analizar la complejidad del proceso de toma de decisiones generado precisamente por la pluralidad de actores (Estado, mercado y sociedad civil) que participan, con intereses distintos y hasta contrapuestos, en el proceso de toma de decisiones. Según ese enfoque, las exigencias funcionales del actual contexto global determinarían la necesidad de transitar desde una noción de gobierno y política pública (como estructura o precondición más rígida y menos flexible), a la noción de gobernanza al momento de elaborar e implementar políticas públicas con impacto sobre el territorio, dejando de lado modelos de imposición.

Desde la óptica de la gobernanza territorial multinivel en clave de desarrollo sostenible, este trabajo analiza la percepción de los actores territoriales acerca de los impactos del cambio climático en una región determinada, así como sus visiones acerca de las políticas y los mecanismos de la gobernanza climática del Uruguay. Se consideran aquí, por un lado, la variable de la articulación entre los diferentes niveles de gobierno y, por el otro, la variable de la participación de distintos actores. Este abordaje puede proporcionar algunas claves relevantes para optimizar un diseño políticoinstitucional que permita potenciar acciones que efectivamente incorporen las visiones de los eventualmente afectados por esta problemática. En ese sentido, no se trata de relevar los impactos reales del fenómeno, sino de identificar las formas como dichos efectos son visualizados (o no) y cómo los actores se posicionan discursivamente frente al fenómeno, desarrollan perspectivas específicas, llegando eventualmente a desarrollar acciones individuales o concertadas. Esto permite, a su vez, identificar clivajes en los respectivos posicionamientos, entendidos como líneas de estructuración de la polarización y la división de posiciones.

La categoría conceptual de "clivajes" fue propuesta por los sociólogos Seymour Lipset y Stein Rokkan (1967) en su análisis sobre los orígenes sociales del sistema de partidos políticos de las sociedades modernizadas, que los autores buscaron explicar identificando las divisorias de confrontación y estructurales que antecedieron y acompańaron ese trayecto modernizador. Al mismo tiempo, su análisis aportaba elementos para una mejor comprensión de los orígenes estructurales de los episodios de conflicto en una sociedad dada -tomando como referencia un nivel intermedio de abstracción-, que se diferenciaban de conflictos reales y concretos, así como configuraciones propias de la socioestructura. Como señala Aguilar (2008), las divisorias que Lipset y Rokkan conceptualizan como clivajes constituyen elementos decisivos de la estructura política de oportunidades que afecta de manera directa 
las motivaciones de los actores (políticos) que intervienen en los conflictos sociales: en ese sentido, un clivaje podría entenderse como una divisoria confrontacional entre grupos de individuos que tiende a organizar los conflictos entre ellos, cuyos efectos no solo se perciben en el sistema político (a través del sistema de partidos, de grupos de interés y otros), sino que afectan o atraviesan todos los componentes estructurales de la sociedad afectada. En América Latina, la categoría se discute con fuerza desde la década de los ochenta, de cara a procesos de democratización, así como en relación con conflictos sociales, político-ideológicos, religiosos o distributivos.

Partiendo de estas categorías analíticas, el presente análisis procura identificar las condiciones que enmarcan procesos colectivos de aprendizaje en diferentes niveles (local, regional y nacional) y que eventualmente permitan superar resistencias frente a la implementación de políticas en esta área. En ese sentido, cabe señalar que la estructura político-administrativa, así como los perfiles económico-productivos y socioculturales de la región Noreste le confieren una especificidad propia y que son relevantes a la hora del diseño e implementación de las políticas públicas departamentales y nacionales. Un reciente estudio de prospectiva territorial sobre esta región (opp, 2017) señaló la necesidad de desarrollar formatos de gobernanza que articulen actores públicos y privados en todos los niveles para lograr implementar políticas públicas efectivas. Se identifican debilidades de la región que obedecen a factores históricos, resaltando las dificultades en el territorio para promover el asociacionismo y generar una visión endógena de sus requerimientos para impulsar procesos de desarrollo. Pese a una coyuntura innovadora (radicación de nuevas inversiones, descentralización universitaria, etc.), la región correría el riesgo de no poder aprovechar su potencial. Consecuentemente, se enfatiza la oportunidad generada por el surgimiento de espacios de articulación interinstitucional y apuestas por esquemas incipientes de gobernanza en términos productivos, pero también sociales y ambientales (Freigedo y Milanesi, 2016).

\section{INSTITUCIONALIDAD DE LA GOBERNANZA CLIMÁTICA EN EL URUGUAY}

Uruguay ratificó la Convención Marco de las Naciones Unidas sobre el Cambio Climático (CMNUCC) de 1992, por la Ley 16.517, de 1994, así como el Protocolo de Kioto (1997), por la Ley 17.279, del año 2000. A nivel nacional, las acciones en torno al fenómeno del cambio climático se enmarcan en la Ley General de Protección del Ambiente (LGPA - 17.283). Dicha Ley, sancionada en 2000, reglamenta a su vez el inciso $1^{\circ}$ del artículo 47 de la Constitución de la República (reformada en 1996), que establece los principios de la política ambiental nacional y sus principales instrumentos de gestión e incorpora al derecho uruguayo el concepto de desarrollo sostenible, como modelo de desarrollo que el Estado deberá propiciar (sNRCC, 2010, p. 30).

Durante el primer gobierno de Tabaré Vázquez se creó, en 2009, el snrcc, que define un esquema institucional que contempla la inclusión de los niveles territoriales a través del Congreso de Intendentes (CI) como actor 
invitado dentro del formato establecido para procurar el desarrollo de la gobernanza climática del país. En consecuencia, dentro del CI se establecen tres lugares para materializar su presencia en el SNRCC, que se distribuyen según la adscripción político-partidaria de las intendencias (figura 1$)^{3}$.

Definido este esquema institucional, desde el SNRCC se hicieron esfuerzos para articular con las intendencias departamentales y por lograr acuerdos interdepartamentales, tales como el caso del área metropolitana con el proyecto Enfoque Territorial del Cambio Climático (TACC, por sus siglas en inglés) (SNRCC, 2014). Asimismo, desde el snrCC, en 2009 se desarrolló una estrategia de consulta e involucramiento de los actores locales realizando una encuesta cerrada y semiestructurada dirigida a los responsables, dentro de los gobiernos departamentales, de la problemática del cambio climático. Entre las situaciones-problemas más nombradas como eventos extremos producidos por la variabilidad climática surgieron la sequía, las inundaciones, así como los temporales, tornados o modificaciones en los patrones de flujo del viento. En relación con la producción agropecuaria, las situaciones-problema más nombradas fueron la escasez de agua para riego, la escasez de agua para animales y los problemas de rentabilidad de los productores (López, 2009). Trabajos posteriores dieron continuidad al estudio de las percepciones de los actores locales de los departamentos de

3 Se define así un representante para el conjunto de intendencias cuyos gobiernos responden al Partido Colorado, otro para las intendencias afines al Partido Nacional y otro para aquellas ligadas al Frente Amplio.
Artigas, Rivera, Cerro Largo y Tacuarembó respecto a los impactos del fenómeno, constatándose la necesidad de definir un adecuado marco político-institucional de articulación que propicie el involucramiento de los actores locales (Stuhldreher, 2016).

En 2010 se hizo público el Plan Nacional de Respuesta al Cambio Climático, diseñado por el SNRCC, que se presentó como el instrumento que el Gobierno nacional y el cr habían establecido para incorporar el fenómeno del cambio climático a la estrategia de desarrollo sostenible a largo plazo del país. Este Plan se señalaba como un vector de importancia para otras áreas de política como la promoción de las zonas rurales, la gestión costera integrada, el ordenamiento del territorio y la conservación y el manejo sostenible de los recursos naturales. Asimismo, se explicitaba la aspiración a conformar un conjunto ordenado de orientaciones $y$ directrices elaboradas en forma interinstitucional y participativa, entendiéndose que la respuesta al cambio climático representaba un desarrollo de largo plazo que debía ser planificado en un proceso iterativo y continuo (sNRCC, 2010).

A partir de 2015, el snRCC se inserta en un esquema más amplio en el marco de la nueva Secretaría Nacional de Ambiente, Agua y Cambio Climático (sNAACC). En 2016, se reglamenta el funcionamiento de esta Secretaría y se establece el Sistema Nacional Ambiental (figura 2).

En 2017 se hizo público el instrumento Política Nacional de Cambio Climático (PNCC), elaborado en forma participativa y multisectorial, que en relación con la gobernanza manifiesta que se deberá garantizar la 
FIGURA 1. ESTRUCTURA DEL SNRCC

\section{SNRCC}

Sistema Nacional de Respuesta al Cambio Climático

Grupo de Coordinación

$1^{\circ}$ Presidente MVOTMA - Ministerio de Vivienda, Ordenamiento Territorial y Medio Ambiente *

$2^{\circ}$ Presidente MGAP - Ministerio de Ganadería, Agricultura y Pesca

$2^{\circ}$ Presidente OPP - Oficina de Planificación y Presupuesto MDN - Ministerio de Defensa Nacional

MEF - Ministerio de Economía y Finanzas

MIEM - Ministerio de Industria, Energía y Minería

MRE - Ministerio de Relaciones Exteriores

MSP - Ministerio de Salud Pública

MINTUR - Ministerio de Turismo

CI - Congreso de Intendentes

SINAE - Sistema Nacional de Emergencias

Invitados: Secretaria de Ambiente, Agua y Cambio Climático

MIDES (Ministerio de Desarrollo Social), Instituto Uruguayo

de Metereología (INUMET) y Agencia Uruguaya de Cooperación Internacional (AUCI)

* El SNRCC es liderado por el Director de la Unidad de Cambio Climático del MVOTMA

\section{Comisión Asesora}

Técnicos de instituciones públicas, entidades académicas técnicas y de investigación

\section{Grupos de trabajo}

80 técnicos de Ministerios, gobiernos departamentales, 80 técnicos de Ministerios, gobiernos departamentales, versidad de la República), INIA (Instituto Nacional de Investigaciones Agropecuarias)

Fuente: elaboración propia.

\section{FIGURA 2. INSERCIÓN DEL SNRCC EN LA SNAACC Y EN EL SISTEMA NACIONAL AMBIENTAL}

Presidencia ROU

República Oriental del Uruguay

\section{SNAACC}

Secretaría Nacional de Ambiente, Agua y Cambio Climático

2015

En 2016 se reglamenta esta Secretaría y se establece

\section{Sistema Nacional Ambiental}

Reúne al Gabinete Nacional Ambiental (GNA)

SNAACC

MIEM, MDN, Secretario Nacional de Ambiente,

OSE (Obras Sanitarias del Estado)

Agua y Cambio Climático

INUMET (Instituto Uruguayo de Metereología)

SNRCC (Sistema Nacional de Respuesta al

Cambio Climático)

SINAE (Sistema Nacional de Emergencias)

Cl (Congreso de Intendentes): invitado 
participación de las instituciones y organizaciones públicas, privadas, académicas y de la sociedad civil, incluyendo expresamente los niveles territoriales. Señala, asimismo, la necesidad de definir espacios interinstitucionales e intersectoriales eficientes que promuevan, formulen, implementen, monitoreen y evalúen la mencionada política y sus líneas de acción, donde el Estado deberá jugar un rol impulsor y conductor de dichos procesos, a través de sus instituciones competentes y en sus distintos niveles de gobierno (sNRCC, 2017a). En el marco de la PNCC, durante 2017 se elaboró la Primera Contribución Determinada a nivel Nacional (NDC, por sus siglas en inglés) bajo el Acuerdo de París, y se presentó ante la Conferencia de las Partes de la CMNUCC ${ }^{4}$.

\section{METODOLOGÍA}

El presente trabajo desarrolló una estrategia metodológica de corte cualitativo, que apuntó a recabar evidencia empírica a través de la revisión bibliográfica y documental (fuentes primarias y secundarias), así como la realización de once entrevistas semiestructuradas a actores de los tres departamentos del Uruguay aquí analizados, tanto del ámbito gubernamental como de la sociedad civil entendida en

4 En la Primera NDC se presentan los objetivos de contribución de mitigación de Uruguay detallados por gas y proyectados al 2025, diferenciando entre objetivos incondicionales y condicionales sujetos a recibir medios de implementación adicionales específicos. Dichos objetivos de mitigación cubren el 99.4\% de las emisiones de gases de efecto invernadero (GEI) del Inventario Nacional de GEI (INGEI) efectuado en 2012 (sNRCC, 2017b). un sentido amplio. Dichas entrevistas fueron efectuadas durante el primer semestre de 2019 y, a través de ellas, se procuró indagar por los posibles clivajes en las percepciones tomando en cuenta las situaciones diferenciales de un amplio espectro de actores radicados en el territorio considerado.

Siguiendo el foco de las preguntas formuladas, es posible agrupar los resultados en tres grandes áreas temáticas: en primer término, se apuntó a generar evidencias acerca del tipo de manifestaciones del fenómeno que se perciben en el territorio considerado, las magnitudes del mismo y la forma en que son percibidas. En segundo lugar, se exploraron las percepciones de los diferentes actores respecto a las políticas y los mecanismos de gobernanza climática desarrollados en el país, en particular respecto a los mecanismos de articulación entre los diferentes niveles de gobierno. En tercer lugar, se indagaron las percepciones sobre las posibilidades de participación por parte de los actores territoriales en los esquemas establecidos de dicha gobernanza: en ese sentido, se tuvieron en cuenta estudios anteriores sobre la historia energética del país, que indican que no hay procesos espontáneos en la dinámica de transformación de la matriz energética, sino que las relaciones sociales, la acción de los grupos de presión, la institucionalidad vigente para la toma de decisiones y el diseño de políticas, así como las propias resistencias sociales ante el cambio constituyen factores que determinarán dicha evolución (Bertoni et al., 2010, p. 187). Eventualmente, factores similares podrían estar afectando las políticas que el país despliega en la actualidad frente al cambio climático, por lo que también se procuró captar las percep- 
ciones en torno de posibles resistencias en esta área temática.

El análisis del discurso desplegado por los entrevistados apuntó a generar evidencia empírica e insumos para una evaluación retrospectiva del diseño y arreglo institucional de la gobernanza climática del país con especial énfasis en el nivel territorial, con la posibilidad de esbozar una proyección a futuro que fortalezca el diseńo y la implementación de políticas públicas asertivas para la región Noreste en materia de cambio climático.

\section{CLIVAJES EN LAS PERCEPCIONES DE LOS ACTORES DEL NORESTE RESPECTO AL CAMBIO CLIMÁTICO}

La presentación de la evidencia empírica y su respectivo análisis se estructura según los focos temáticos mencionados, sistematizándose las percepciones diferenciales de los informantes calificados de los tres departamentos. Esto incluye, por un lado, a actores del ámbito gubernamental, tanto de representantes a nivel departamental de las oficinas de Desarrollo y Medioambiente u órganos similares de las respectivas intendencias, así como de representantes de organismos como ministerios nacionales con representación territorial. Por otra parte, se consideran también las visiones de actores de la sociedad civil, tales como grandes y pequeńos productores o sus respectivas agremiaciones, academia, organizaciones sociales y organizaciones no gubernamentales (ONG).

\section{Percepciones sobre el alcance y la magnitud del fenómeno: identificación de problemáticas actuales}

En primer lugar, se indagó acerca de las manifestaciones del fenómeno tal como se perciben en el territorio y las magnitudes del mismo. La gran mayoría de los representantes se remite a la evidencia científica sobre el fenómeno y coincide en su carácter antropogénico. Una excepción la constituye el representante de la Dirección de Ordenamiento Territorial de la Intendencia de Tacuarembó (IDT) que plantea dudas respecto a si efectivamente el hecho analizado tiene entidad real: "No me queda claro que exista el cambio climático o no. Algunos meteorólogos y científicos dicen que estos son ciclos que se dan cada tanto. Hay un negocio detrás de esto" ${ }^{5}$. En otro sentido, representantes de la intendencia departamental de Cerro Largo alertan contra el tremendismo y vinculan el cambio climático a un fenómeno natural del planeta, subrayando la capacidad para lidiar con el proceso actual: "Es un tema muy vasto, lamentablemente opina mucha gente sin saber, opinando lo que es políticamente correcto [...]. Este es un fenómeno natural del planeta, es el primer cambio climático generado por humanos [...]. Tenemos la capacidad de lidiar con el cambio climático actual"6.

En lo tocante a los eventos más acuciantes, numerosos representantes confirman en

5 Entrevista a Walter Mederos, director de Ordenamiento Territorial de la IDT, 19 de marzo de 2019.

6 Entrevista a al ingeniero Javier Pena Venturiello, representante técnico de proyectos de la Secretaría Ejecutiva de la Intendencia de Cerro Largo, 12 de junio de 2019. 
la actualidad aquellos tópicos que habían surgido en el estudio efectuado por el SNRCC entre septiembre y noviembre de 2009, donde los eventos más nombrados fueron la sequía y las inundaciones, así como los temporales y tornados. Junto con esta confirmación de lo acuciante de los sucesos por parte de representantes del sector productivo forestal ("Lo que preocupa es la impredecibilidad y lo dramático de los eventos") ${ }^{7}$, para un representante de la academia radicado en la Estación Experimental Bernardo Rosengurt (EEBR) de la Universidad de la República (Udelar) en el departamento de Cerro Largo, estos eventos se insertan en un patrón histórico propio del Uruguay: "Sequías, inundaciones y tornados fueron siempre parte de este país. De lo que se trata ahora es de determinar la frecuencia e intensidad de los eventos extremos" ${ }^{8}$. Por otra parte, varios actores mencionan otras manifestaciones especialmente preocupantes del fenómeno: así, representantes de la Dirección de Desarrollo Rural del MGaP radicada en Tacuarembó mencionan el recrudecimiento de las granizadas ${ }^{9}$. Como actor de la sociedad civil en los departamentos de Rivera y Tacuarembó, un representante de la Fundación INDRA suma el riesgo de incendios en un área con extensas plantaciones forestales.

7 Entrevista al ingeniero agrónomo Juan Pedro Posse, gerente de I\&D de la empresa forestal LUMIN y representante de la Sociedad de Productores Forestales (SPF) del Uruguay, 17 de mayo de 2019.

8 Entrevista al ingeniero agrónomo Carlos Mantero, director de la EEBR/UDELAR, 11 de abril de 2019.

9 Entrevista a las ingenieras agrónomas Mercedes Figari y Mariana Brunel, Dirección de Desarrollo Rural del MGAP en Tacuarembó, 22 de marzo de 2019.
Desde una mirada diferente, la representante de Medioambiente de la IDT subraya la necesidad de conectar la percepción del fenómeno del cambio climático con prácticas determinadas por situaciones de pobreza: "Es el caso del productor que ara a favor de la pendiente para que no se le pudra la papa. En la gestión de los residuos lo veo también" ${ }^{10}$. A su vez, el director del Instituto Nacional de Investigaciones Agropecuarias (INIA), radicado en Tacuarembó, expresa que entre las problemáticas más acuciantes para el sector agropecuario debería incluirse el impacto sobre los recursos naturales (como el suelo), a la vez que señala la necesidad de concientización respecto de las implicancias de los compromisos internacionales como el Acuerdo de París, que en la actualidad generan una contextualización diferente a la que existía en el momento de la consulta de 2009: "Uruguay generó un compromiso con el mundo: es un gran desafío. Después está la cuestión de si lo cajoneamos"11.

Asimismo, desde ámbitos de investigación como el Programa Forestal del INIA se señala que más allá de los eventos extremos más acuciantes, existen toda una serie de impactos del cambio climático que son más de largo plazo: "Las especies forestales y agrícolas están adaptadas a cierta temperatura, a cierto régimen hídrico, todo con años de mejoramiento. A lo que voy es a la necesidad de adaptación de esos sistemas productivos, que ya se están empezando a ver, hay diferencias

10 Entrevista a la ingeniera agrónoma Laura Lacuague, representante de Medioambiente de la IDT, 19 de marzo de 2019.

11 Entrevista al doctor Fabio Montossi, director Nacional del INIA, 1 de abril de 2019. 
en los ataques de plagas, una tropicalización de las enfermedades" ${ }^{\prime 2}$. Al mismo tiempo, se mencionan eventuales impactos favorables en algunos sectores productivos, como el caso de la especie introducida eucaliptus grandis, que en Uruguay se ubica en el límite de frío de la especie: con el aumento de lluvias y de temperaturas disminuye el periodo de la edad de corte. Asimismo, se menciona una modificación de la estructura y calidad de la madera, que mejora en el caso del eucaliptus, mientras que empeora en el caso del pino (la otra especie introducida con fuerte presencia en las plantaciones comerciales de la región Noreste), por el aumento del grosor de los anillos de crecimiento. En un sentido similar, desde la División de Medioambiente de la intendencia departamental de Rivera (IDR) también se expresa preocupación por la tropicalización del clima y sus impactos en cultivos característicos de la región como la sandía, y en las plantaciones forestales. Junto con la preocupación por los impactos de la tropicalización en términos de la salud humana (con el ejemplo de la adaptación del mosquito transmisor del dengue), se hace referencia al aumento del uso de agroquímicos para combatir las plagas: "Nosotros en la IDR trabajamos con el programa 'Campo Limpio', que recolecta y recicla los recipientes de agroquímicos. Con base en eso tenemos la percepción de cómo aumenta la venta de esos productos, más allá de las consideraciones ambientales. Se perciben más plagas. Hay figuras nuevas, al no haber más inviernos rigurosos"13.

12 Entrevista a la doctora Cecilia Rachid, investigadora del Programa Forestal del INIA, 10 de abril de 2019.

13 Entrevista a José Almada Sad, director de la División de Medioambiente de la idr, 3 de junio de 2019.

\section{Percepción sobre políticas y mecanismos de gobernanza climática: articulación entre diferentes niveles de gobierno}

Consultados los representantes radicados en el territorio acerca de su percepción acerca de las políticas de cambio climático definidas para el país, desde la dirección de la EEBR se menciona la fragmentación del trabajo de los organismos gubernamentales que debieran estar mejor articulados en torno a esta problemática, aunque se valore como importante la definición de políticas públicas en este tema desde el primer gobierno de Tabaré Vázquez: "Marcar una dirección y avanzar en ese sentido" ${ }^{14}$. También, desde la dirección de INIA -a la vez de señalar algunas disputas desde el punto de vista de las intervenciones de cada ministerio dentro del SNRCC- se hace referencia a la necesidad de optimizar la coordinación de las acciones: "Los mecanismos de gobernanza, liderazgo, priorización marcan claramente la impronta de cada espacio. A pesar de los avances veo que todavía seguimos siendo un gran archipiélago de instituciones" ${ }^{15}$. En particular, diversos representantes mencionan disputas entre el mvotma y el mgap. Por otra parte, desde distintos ámbitos nacionales como el MGAP en su representación territorial se subraya el desconocimiento respecto de los lineamientos centrales de la Política Nacional y del Plan Nacional de Respuesta al Cambio Climático: "No hay mucha claridad respecto a cuál es la vinculación de esto que hacemos con el Plan

14 Entrevista al ingeniero agrónomo Carlos Mantero, director de la EEBR/uDELAR, 11 de abril de 2019.

15 Entrevista al doctor Fabio Montossi, director nacional del INIA, 1 de abril de 2019. 
Nacional de Respuesta al Cambio Climático. Nadie me invitó a leerlo"16. Desde una visión crítica, el representante de Ordenamiento Territorial de Tacuarembó atribuye escasa incidencia de los lineamientos mencionados en las políticas sectoriales ${ }^{17}$. En contraste con eso, desde ámbitos de investigación como INIA se indica que sí ha permeado y se han cristalizado en líneas de trabajo, aunque cabría fortalecer la transversalidad entre todos los programas ${ }^{18}$.

Respecto a la inserción del SNRCC a partir de 2015 en un esquema más amplio en el marco de la nueva Secretaría Nacional de Ambiente, Agua y Cambio Climático (sNAACC), algunos representantes de la intendencia de Tacuarembó interpretan ese paso como un proceso de recentralización del poder, ya que la mencionada Secretaría respondería directamente a la Presidencia de la República ${ }^{19}$. En un sentido similar, desde espacios con fluida vinculación con el SNRCC, como la Dirección de Medioambiente de la IDR, se coincide en el diagnóstico: "Es un secreto a voces de que se creó esta Secretaría para que dependiera directamente de Presidencia. Quizás hubo una visión de que el SNRCC era muy autónomo. No sé si necesariamente fue para 'disciplinar' al SNRCC, pero sí para que la Presidencia tuviera un interlocutor en el medio. Pero la Secretaría

16 Entrevistas a la ingeniera agrónoma Mercedes Figari y Mariana Brunel, Dirección de Desarrollo Rural del MGAP en Tacuarembó, 22 de marzo de 2019.

17 Entrevista a Walter Mederos, director de Ordenamiento Territorial de la IDT, 19 de marzo de 2019.

18 Entrevista a la doctora Cecilia Rachid, investigadora del Programa Forestal del INIA, 10 de abril de 2019.

19 Entrevista a Walter Mederos, director de Ordenamiento Territorial de la IDT, 19 de marzo de 2019. no incide, no mueve la aguja. Quizás en el futuro se fortalece" ${ }^{20}$. Por otra parte, desde la sociedad civil se subraya que no se visualizan tensiones entre la Secretaría y el SNRCC, señalándose diferencias en los roles diferenciales de cada uno: "Lo que hace el SNRCC es mucho más a terreno, mucho más operativo con nosotros. La Secretaría cumple una función más bien de articulación con los públicos, no con el territorio" ${ }^{21}$.

Por otra parte, el análisis de las percepciones sobre los mecanismos de la gobernanza climática y sobre la articulación entre diferentes niveles de gobierno presenta clivajes claros dependiendo de los actores consultados. Cabe señalar el posicionamiento diferencial que asume el departamento de Rivera, que desde hace veinte años procura desplegar un conjunto de políticas ambientales en sentido general y algunas acciones específicas en materia de cambio climático, donde en algunos casos se procuró trabajar en clave de cooperación territorial con otras intendencias ${ }^{22}$, destacando en particular los recientes avances para el Plan Departamental de Adaptación al

20 Entrevista a José Almada Sad, director de la División de Medioambiente de la IDR, 3 de junio de 2019.

21 Entrevista al ingeniero agrónomo Aler Donadio, Fundación INDRA, 3 de junio de 2019.

22 Puede mencionarse el impulso de la IDR para la realización en 2012 de pasantías y visitas a cuatro provincias y seis ciudades de Argentina, y los contactos con la Red Argentina de Municipios contra el Cambio Climático (RAMCC), que se hizo extensivo a las intendencias del norte (participaron Artigas, Tacuarembó, Cerro Largo y Salto). También se lideró un viaje a Bolivia coordinado con la Fundación Nativa a fin de conocer iniciativas sobre adaptación al cambio climático, con invitación a las intendencias del norte (participó Salto). 
Cambio Climático para Rivera y Tacuarembó, que se presentaron en mayo de 2019. Por otra parte, la IDR ocupa uno de los tres lugares definidos por el cr para el nivel departamental en el marco del SNRCC, específicamente el que corresponde a intendencias bajo la égida del Partido Colorado. En ese contexto, actores vinculados a la División de Medioambiente de la intendencia subrayan lo fluido de los vínculos establecidos entre el nivel departamental y el SNRCC; se hace así referencia a un proceso de construcción de lazos de confianza mutua donde no surgieron rispideces ideológicas: "Esta relación institucional se construyó muy bien, felizmente. Nunca hubo una traba por cuestiones político-partidarias. Tampoco con otras intendencias de otros partidos. Institucionalmente la relación es muy fuerte entre IDR y snRCC, y también con la Oficina de Planificación y Presupuesto (OPP) con temas de reciclado. Es muy creíble la relación"23. Por su parte, representantes de la sociedad civil que actúan en varios departamentos de la región, como la Fundación INDRA, señalan las diferencias entre los casos de Rivera y Tacuarembó: "En Rivera hay muy buena articulación en términos de la gobernanza entre lo local y lo nacional. Es un caso para aprender. Si bien hay orientaciones político-partidarias diferentes, hay cantidad de proyectos con los que desde Rivera se articula con el gobierno nacional. En clima y en otras áreas" 24 .

23 Entrevista a José Almada Sad, director de la División de Medioambiente de la IDR, 3 de junio de 2019.

24 Entrevista al ingeniero agrónomo Aler Donadio, Fundación INDRA, 3 de junio de 2019.
Aunque se mencionan algunas dificultades para conciliar tiempos diferentes entre las respectivas intendencias y para acceder a la información incluso de organismos públicos como MGAP o Instituto Uruguayo de Meteorología (INUMET), los representantes de Rivera destacan en clave de articulación territorial como resultado significativo el mencionado Plan de Adaptación para Rivera y Tacuarembó, diseñado en un proceso consultivo entre 2017 y 2019 a través de talleres realizados en ambos departamentos con actores diversos y contando con financiamiento de la Agencia Española de Cooperación para el Desarrollo (AECID) para los gastos operativos, así como con asesoramiento técnico de la Fundación Nativa (Bolivia) y la Fundación InDRA: "Llegamos a esta herramienta, que es la vedette pero no es el fin de nada" 25 . De hecho, en el resumen ejecutivo se plantea explícitamente la conexión con el nivel nacional a través del Plan Nacional de Respuesta al Cambio Climático de 2010, así como la proyección a futuro:

Esta iniciativa busca llevar esta política nacional a nivel de la política departamental de las Intendencias de Rivera y Tacuarembó, con el objetivo de reducir la vulnerabilidad de los entornos urbanos y rurales al 'cambio climático y la variabilidad' (CCV), asociadas a las principales amenazas identificadas. Este Plan pretende ser el inicio de un proceso a mediano y largo plazo que contribuya al desarrollo de las capacidades locales para la mitigación y adaptación en un marco general del desarrollo sostenible. (López y Larghero, 2019, p. 2)

Por otra parte, también debe mencionarse la participación a futuro del departamento de

25 Entrevista a José Almada Sad, director de la División de Medioambiente de la IDR, 3 de junio de 2019. 
Rivera como única intendencia del norte en el proyecto NAP Ciudades ${ }^{26}$ y en el proyecto de Movilidad Urbana Sostenible impulsado por el Ministerio de Industria, Energía y Minería (мIEм), el mvotma, el Ministerio de Transporte y Obras Públicas (MTOP) y el Ministerio de Economía y Finanzas (MEF) y que apunta a la incorporación de ómnibus y taxis eléctricos ${ }^{27}$.

Por su parte, desde la oficina de Proyectos de Desarrollo y Medioambiente (PRODEMA), de la intendencia de Tacuarembó, la representante de Medioambiente hace mención de las debilidades de articulación departamental con el nivel nacional: "Están más distanciadas hoy que cuando surgió el SNRCC. Se dieron acciones desarticuladas de la planificación que se hizo [...] No toda la responsabilidad es del SNRCC" 28 . Se señala la necesidad de que desde los niveles departamentales se trabaje con mayor fuerza, así como de que se fortalezca la articulación entre departamentos de la misma región, por ejemplo, de cara a la implementación del mencionado Plan de Adaptación, ya que se considera que hubo escasa interac-

26 En el marco del Plan Nacional de Adaptación al Cambio Climático, el proyecto NAP Ciudades constituye una iniciativa que apunta a propiciar la adaptación al cambio climático en ciudades e infraestructuras. Está financiado por una donación del Programa Preparatorio del Fondo Verde para el Clima (FvC) establecido en la Cumbre de París, y es gestionado por el Programa de Naciones Unidas para el Desarrollo (PNUD).

27 El proyecto se llevará a cabo en el marco de Euroclima+ (Unión Europea), que apoya la implementación de acciones que contribuyan a cumplir con el Acuerdo de París.

28 Entrevista a la ingeniera agrónoma Laura Lacuague, representante de Medioambiente de la IDT, 19 de marzo de 2019. ción previa. Con preocupación se subraya la escasa percepción de los actores locales frente a la problemática del cambio climático: "En el proyecto de prospectiva territorial 'Visión 2050: Tacuarembó en la región Norte' ${ }^{29}$ en la consulta con actores no surgió el cambio climático como desafío. Indica que no se está haciendo una buena labor"30.

Desde un organismo nacional con presencia territorial, como la Dirección de Desarrollo Rural del MGAP, también se constatan desafíos de articulación entre diferentes niveles de actuación en materia de políticas referidas al cambio climático, incluso dentro del mismo ministerio. En ese sentido, se hace referencia al proyecto "Ganaderos familiares y cambio climático" (GFCC), cuyo anclaje territorial refiere a algunas secciones de los departamentos de Salto, Artigas, Tacuarembó, Rivera y Paysandú, y cuyo objetivo central consiste en fortalecer la resiliencia de los territorios frente al fenómeno. Mientras que el esfuerzo específico en materia de cambio climático en el primer componente del mencionado proyecto permitió efectivamente poner medios a disposición de los productores para reducir su vulnerabilidad, en el segundo componente se trabajó más bien en un necesario prerrequisito: fortalecer las redes propias del espacio rural, como las Mesas de Desarrollo Rural. Finalmente, en el tercer com-

29 El mencionado proyecto de prospectiva territorial fue realizado entre 2016 y 2017 por equipos locales de la UDELAR en conjunto con la IDT, el INIA, la Mesa de Desarrollo Local (MDL) y la opp, lo que implicó un amplio proceso de consulta participativa a actores de Tacuarembó y Rivera.

30 Entrevista a la ingeniera agrónoma Laura Lacuague, representante de Medioambiente de la IDT, 19 de marzo de 2019. 
ponente referido a la gestión del conocimiento se apuntó a generar información vinculada a la reducción de los impactos del cambio climático, a través de un convenio con la Facultad de Agronomía de la udelar basado en principios de coinnovación. Al momento de la culminación del proyecto la evaluación es mixta: "Se puso mucho esfuerzo en el componente 1, los otros estaban más desdibujados. Los fondos del GFCC no los maneja en su totalidad nuestra Dirección [...]. Es como muy desdibujada la gestión del programa en su conjunto [...]. Ha habido una vinculación entre los territorios y el nivel central dentro del MGAP, que es como heterogénea" ${ }^{31}$. Más allá de disponer de un enfoque orgánico en la aplicación de las políticas dentro de un mismo ministerio ("esta es una herramienta que nosotros bajamos a nuestro territorio, pero no es una herramienta de nuestra Dirección. Hay problemas de organigrama, de estructura, que atraviesan todo"), también se subraya la falta de conciencia respecto a la importancia del fenómeno, así como la complejidad del lenguaje técnico utilizado en torno a la problemática: "Tampoco es una prioridad para la gente. Recién ahora se está empezando a entender qué es. Con los productores hay un problema de conceptos que no es fácil transmitir. 'Mitigar', 'resiliencia', no está traducido en lenguaje coloquial" 32 .

31 Entrevistas a las ingenieras agrónomas Mercedes Figari y Mariana Brunel, Dirección de Desarrollo Rural del MGAP en Tacuarembó, 22 de marzo de 2019.

32 Idem.

\section{Percepciones sobre la participación del territorio en la gobernanza climática e identificación de eventuales resistencias}

Respecto a las posibilidades de participación del territorio en el esquema de gobernanza climática desde la Dirección de INIA se reconocen debilidades, donde los grandes ausentes serían los actores locales, y donde se visualizan ciertas asimetrías en el territorio. En esa línea de argumentación, se otorga a la investigación -en particular aquella realizada en el territorio- un rol que podría propender a superar dichas brechas: "Desde la Ciencia y Tecnología se podría contribuir en el empoderamiento de mecanismos que fortalezcan a los actores locales. Me pregunto: ¿qué herramientas tienen hoy las Intendencias para poder tener una política para el departamento?; ¡cómo aterriza la política de cambio climático en el departamento; ¿cómo discuten con los actores nacionales? [...] Acá en estos temas se necesitan núcleos críticos fuertes" ${ }^{\text {"33 }}$. Otros investigadores de INIA señalan la posibilidad de optimizar la participación de la academia en el SNRCC $^{34}$, más allá del aporte de la información proporcionada por la investigación de la Unidad de Agroclima y Sistemas de Información (GRAS) de INIA desde hace veinte años ${ }^{35}$. Dicha Unidad, de

33 Entrevista al doctor Fabio Montossi, director Nacional del INIA, 1 de abril de 2019.

34 Entrevista a la doctora Cecilia Rachid, investigadora del Programa Forestal del INIA, 10 de abril de 2019.

35 Tras la adhesión de Uruguay a la CMNUCC se crea la Comisión de Cambio Global a nivel nacional. En 1998, se establece en la Estación Regional La Estanzuela de INIA un grupo técnico para realizar estudios sobre el clima y el cambio climático con énfasis en la implicancia sobre la actividad agropecuaria. En 2003, la Junta Directiva de 
carácter nacional, en asociación con equipos interdisciplinarios internacionales, tiene como cometido central la ejecución de proyectos y la elaboración de productos tecnológicos para el desarrollo de Sistemas de Información y Soporte para la Toma de Decisiones (sISTD), focalizando la gestión (prevención y manejo) de los riesgos asociados al clima en la producción agropecuaria. Se procura integrar aquí herramientas como la teledetección, los sistemas de información geográfica (sIG), los sistemas de posicionamiento global (GPS) y los modelos de simulación, para evaluar y determinar el impacto y la vulnerabilidad al cambio climático de rubros y sistemas de producción agropecuarios y forestales, identificando eventuales medidas de adaptación (INIA, 2018).

Concordante con esta visión crítica, según el director de la EEBR, la institucionalidad definida no siempre permite la retroalimentación fluida de visiones y la transmisión de demandas ${ }^{36}$. También, desde la sociedad civil se expresa la falta de espacios para su participación: "Hay pocos espacios. Yo creo que es una política donde prima lo estatal. Donde la fortaleza son los propios organismos del Estado [...] Hay además desactualización en lo que hace a la convocatoria a la sociedad civil: faltan criterios claros para definir quién participa"37.

Específicamente, desde los niveles de algunas intendencias como la de Cerro Largo se plantea el desafío de la lejanía y las distancias

INIA formaliza la creación de la Unidad de Agroclima y Sistemas de Información (GRAS) (INIA, 2018).

36 Entrevista al ingeniero agrónomo Carlos Mantero, Director de la EEBR/UDELAR, 11 de abril de 2019.

37 Entrevista al ingeniero agrónomo Aler Donadio, Fundación INDRA, 3 de junio de 2019. geográficas para mantener la participación en las convocatorias y reuniones que, en su mayoría, se realizan en la capital, así como la necesidad de optimizar un manejo más sistemático de la información ${ }^{38}$. En contraste con ello, desde otras intendencias como Rivera se procura apostar a desarrollar presencia en esos ámbitos: "Debo decir que tratamos de participar mucho. ¿Somos escuchados? Sí, aunque no siempre es fácil influir con nuestras posturas locales"39.

Consultados respecto a eventuales posturas que expresen resistencia a la implementación de políticas de cambio climático en el territorio considerado, resultan llamativas las consideraciones unánimes que no identifican posicionamientos explícitamente adversos en esta materia. Aunque se resaltan ausencias significativas del fenómeno en proyecciones a futuro, como en el mencionado ejercicio de prospectiva territorial efectuado en Tacuarembó con inclusión del área regional de Rivera ${ }^{40}$, varios representantes de distintos ámbitos coinciden en que aún existe gran desconocimiento sobre el fenómeno y sus implicancias, así como de los mecanismos de la gobernanza climática definidos para el país. De esta manera, representantes de la agremiación de los productores forestales señalan: "Este esquema

38 Entrevista al ingeniero agrónomo Javier Pena Venturiello, representante técnico de proyectos de la Secretaría Ejecutiva de la Intendencia de Cerro Largo, 12 de junio de 2019.

39 Entrevista a José Almada Sad, Director de la División de Medioambiente de la IDR, 3 de junio de 2019.

40 Entrevista a la ingeniera agrónoma Laura Lacuague, representante de Medioambiente de la IDT, 19 de junio de 2019. 
de gobernanza es bastante desconocido entre los privados [...] Hay escasa participación pero resistencias no hay. No se prioriza esto en la agenda, por eso quizás hay reticencia para invitaciones. Pero si se logra llegar con buena información, existiría posibilidad de lograr buenos planes de trabajo" ${ }^{41}$.

En la Dirección de la EEBR tampoco se identifican resistencias, a la vez que se señala que para el sector privado existirían incentivos para acciones proclives a la mitigación, como el proceso de certificación, por ejemplo, del sector forestal, a fin de poder seguir adelante con el negocio y la explotación, lo que a futuro tendría un proceso similar para el sector ganadero $^{42}$. Tampoco desde los ámbitos gubernamentales, como el MGAP, se identifica oposición a acciones relativas al cambio climático por parte de pequeńos productores: "No, de eso no vemos. Las cosas que se hacen para mitigar además mejoran la productividad. Es ganar-ganar. El tema es que te crean. Las cosas los productores las hacen desde hace tiempo porque tienen una lógica ancestral" ${ }^{43}$. En un sentido similar, desde la IDR no se visualiza obstrucción a estas actividades: "No, honestamente, no, más bien lo contrario: mucho interés del sector forestal, de pequeños productores

41 Entrevista al ingeniero agrónomo Juan Pedro Posse, gerente de I\&D de la empresa forestal LUMIN y representante de la Sociedad de Productores Forestales (SPF) del Uruguay, 17 de mayo de 2019.

42 Entrevista al ingeniero agrónomo Carlos Mantero, director de la EEBR/UDELAR, 11 de abril de 2019.

43 Entrevista a las ingenieras agrónomas Mercedes Figari y Mariana Brunel, Dirección de Desarrollo Rural del MGAP en Tacuarembó, 22 de marzo de 2019. rurales. Suena a veces como tema lejano, pero nadie se resiste" ${ }^{44}$.

\section{CONCLUSIONES}

Después de diez años de funcionamiento del SNRCC surge la necesidad de una evaluación retrospectiva, vinculada con la posibilidad de optimizar el diseño e implementación de políticas que salgan al encuentro de los desafíos climáticos y profundicen las acciones en curso. Entendiendo que el éxito de esas políticas se ve en gran medida afectado por las visiones de los actores locales y su disposición al compromiso con las estrategias pergeñadas en el nivel nacional, el presente estudio de caso, de corte cualitativo, indaga acerca de las percepciones de los actores territoriales de la región Noreste del Uruguay respecto a tres ejes temáticos, donde se identifican coincidencias y clivajes que se sistematizan a continuación.

En primer término, en relación con el alcance y la magnitud del fenómeno, los actores coinciden -con algunas excepciones-en señalar el carácter antropogénico del cambio climático. Es significativo que dentro de una región compuesta por tres departamentos se identifiquen posicionamientos que van desde falta de certeza respecto a la existencia real del fenómeno, hasta posturas sumamente activas en la búsqueda de soluciones, con posiciones intermedias que relacionan las discusiones actuales con modas discursivas, donde el fenómeno se vincularía más bien al calentamiento

44 Entrevista a José Almada Sad, director de la División de Medioambiente de la IDR, 3 de junio de 2019. 
"natural" del planeta. Por otra parte, existen coincidencias en lo relativo a que los factores más acuciantes identificados en la consulta efectuada por el SNRCC en 2009 (sequías, inundaciones, así como temporales y tornados) continúan vigentes. Como manifestaciones más recientes se agregan consideraciones respecto al aumento de la crudeza de las granizadas, la tropicalización de las enfermedades y plagas, el creciente riesgo de incendios, así como la explicitación de los vínculos del cambio climático con la pobreza.

En segundo término -vinculado con la percepción de los mecanismos de gobernanza climática del país y la articulación entre diferentes niveles de gobierno- es evidente que en la región aún existe un notorio desconocimiento respecto al esquema institucional de gobernanza y las políticas climáticas definidas para el país, incluso dentro de ámbitos gubernamentales nacionales con presencia en el territorio. En ámbitos ligados a la investigación, así como en ámbitos privados, se percibe a dichos mecanismos como una institucionalidad fragmentada, donde se hacen presentes tensiones e intereses ministeriales heterogéneos. Respecto a la valoración de la articulación entre diferentes niveles, se percibe un fuerte clivaje entre las percepciones diferentes de representantes de distintos gobiernos departamentales: por un lado, se da el caso de la intendencia de Rivera, que durante veinte años buscó impulsar políticas ambientales a nivel departamental, liderando la redacción del Plan de Adaptación al Cambio Climático para Rivera y Tacuarembó y que -independientemente de los colores partidarios- percibe favorablemente las posibilidades de articulación con el nivel nacional y que apunta a dar continuidad a su presencia en actividades y mecanismos del SNRCC. En contraste con eso, representantes de intendencias como Tacuarembó o Cerro Largo se muestran más críticos de la institucionalidad en materia climática, pese a su menor grado de interacción con la misma. A su vez, desde la sociedad civil se señalan las diferencias en el accionar de dichos gobiernos departamentales a la hora de desarrollar acciones en materia ambiental o, específicamente, en relación con el cambio climático.

En tercer término -en relación con la participación del territorio en la gobernanza climática y eventuales resistencias-, los actores consultados, en forma casi unánime, expresan una fuerte preocupación por la debilidad de la representación de los niveles territoriales en los mecanismos de la gobernanza climática definida para el país, en particular dentro del SNRCC. Desde la academia se visualizan las asimetrías del territorio, y se expresa preocupación por las formas en que los aportes de la ciencia y tecnología debieran tender a superar las brechas existentes. Por su parte, representantes de las agremiaciones privadas señalan que el desconocimiento de los mecanismos no contribuye a generar interés en la participación. En contraste con esto, desde la sociedad civil se señala la necesidad de establecer criterios claros para definir su participación en los mecanismos del SNRCC. Finalmente, en lo tocante a las eventuales resistencias, resulta relevante destacar que ninguno de los representantes entrevistados identificó posturas abiertamente opuestas a las políticas o acciones en materia de cambio climático. Por el contrario, algunos actores del ámbito privado destacan que de mejorar 
FIGURA 3. CLIVAJES EN LAS PERCEPCIONES DE LOS ACTORES TERRITORIALES DE LA REGIÓN NORESTE

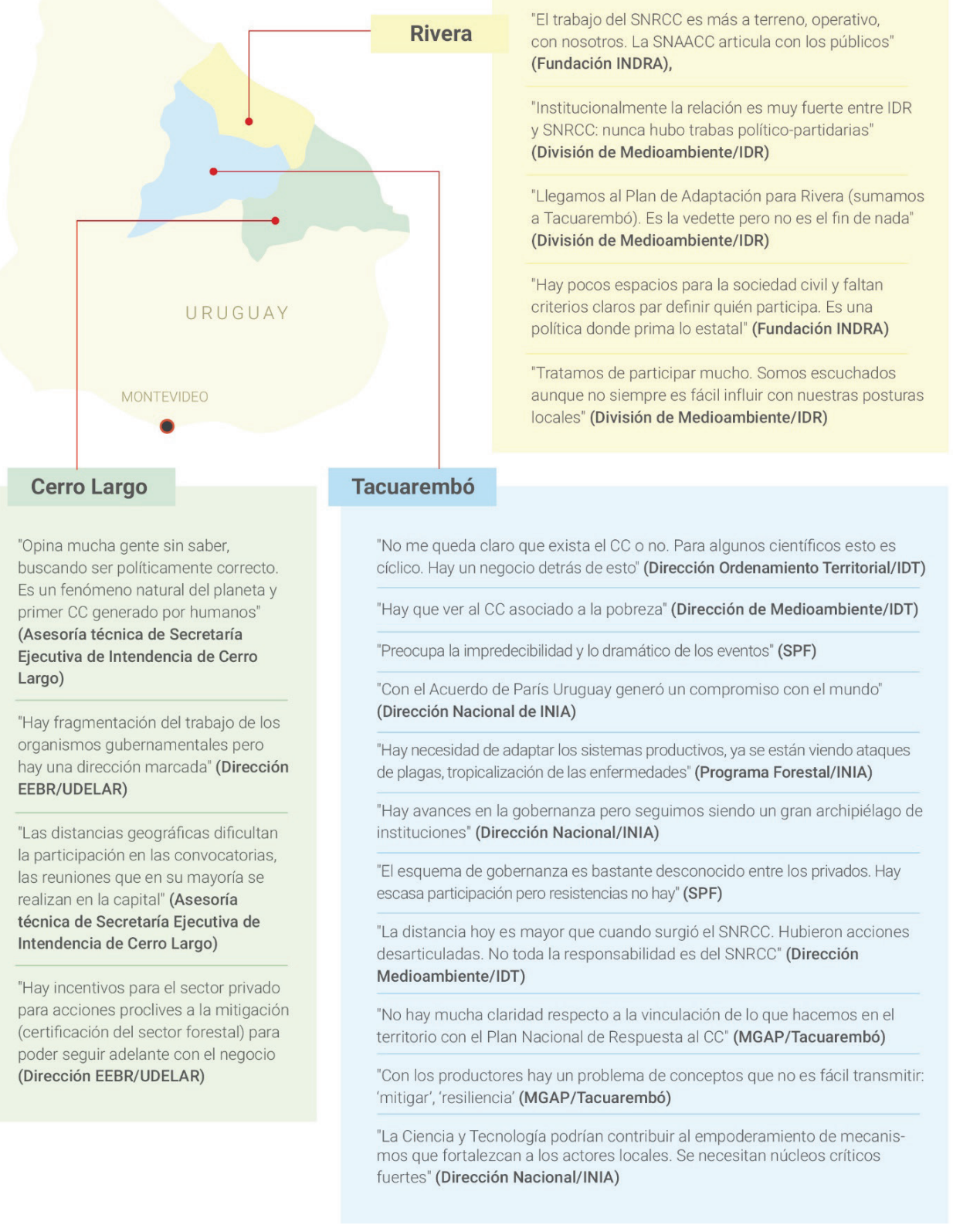

Fuente: elaboración propia. 
el flujo de información podrían superarse algunas reticencias a la participación. En forma similar, desde ámbitos gubernamentales, tanto del nivel nacional como departamental, se señala la necesidad de mejorar la comunicación y el acceso a la información como estrategia para optimizar la convocatoria y la credibilidad de las políticas relativas al cambio climático.

Finalmente, más allá de las connotaciones locales del presente análisis, es posible identificar elementos relevantes para la consideración de otras experiencias. Así, los resultados del estudio muestran la importancia estratégica de profundizar acciones de concientización respecto a la problemática del cambio climático como condición necesaria para el éxito de las estrategias adaptativas o de mitigación. Asimismo, este análisis muestra que en términos del diseño de políticas y mecanismos de gobernanza es crucial superar las tensiones entre diferentes ministerios y dependencias, que determinan la implementación fragmentaria de las iniciativas. En el caso analizado, pese a los avances notorios, en el SNRCC se evidencia esta hipoteca, que afecta las posibilidades de generar un marco eficaz y eficiente para una gobernanza ambiental. También, el centralismo que marca muchos países latinoamericanos es un factor que se debe considerar. De cara a los territorios del interior del Uruguay es notoria la necesidad de dar mayor visibilidad al esquema de gobernanza climática. Resulta aquí estratégico intensificar el involucramiento de los territorios en los mencionados mecanismos, apuntando a un real empoderamiento de los espacios definidos para los niveles subnacionales de gobierno, así como para la sociedad civil, la academia y el sector privado. Al mismo tiempo, es pertinente revisar las capacidades institucionales de los territorios para enfrentar los desafíos climáticos, donde las instituciones abocadas a la investigación radicadas en el territorio deberían asumir un rol más asertivo. A su vez, la aplicación de esas políticas demanda una participación más activa de la ciudadanía, ya que la conciencia social sobre la urgencia del fenómeno constituye un catalizador crucial en la estrategia climática del país.

\section{REFERENCIAS}

Aguilar, S. (2008). La teoría de los clivajes y el conflicto social moderno. Ponencia presentada en noviembre de 2008 al Congreso de Ciencia Política Crítica, Universidad del País Vasco (UPV), Bilbao. Recuperado de http://diposit.ub.edu/dspace/ handle/2445/11012

Basco, A. I. (2018): Medio ambiente y cambio climático. Ideas de integración, 257. Recuperado de http://conexionintal.iadb.org/2018/01/29/ medio-ambiente-cambio-climatico/?utm source=Español\&utm_campaign=99818b6ff9EMAIL_CAMPAIGN_2017_11_09\&utm_ medium=email\&utm_term=0_a72951704999818b6ff9-161085021" http://conexionintal.iadb.org/2018/01/ HYPERLINK "http://conexionintal.iadb.org/2018/01/29/ medio-ambiente-cambio-climatico/?utm source=Español\&utm_campaign=99818b6ff9EMAIL_CAMPAIGN_2017_11_09\&utm _ medium=email\&utm_term=0_a72951704999818b6ff9-161085021”29/medio-ambiente-cambio-climatico/?utm_source= Espa\%C3\%B1ol\&utm_campaign=99818b6ff9EMAIL_CAMPAIGN_2017_11_09\&utm_ 
medium=email\&utm_term=0_a72951704999818b6ff9-161085021

Bertoni, R., Echinope, V., Gaudioso, R., Laureiro, R., Loustaunau, M. y Taks J. (2010). La matrizenergética: una construcción social (Colección Art. 2). Montevideo: CSIC, UDELAR.

Carmona, R. (2006). Nuevas formas de gobierno y gestión pública en el escenario local: elementos y perspectivas para el estimulo de procesos concertados de desarrollo. Buenos Aires: Universidad Nacional General Sarmiento.

Comisión Económica para América Latina y el Caribe (CEPAL) (2014). La economía del cambio climático en América Latina y el Caribe. Paradojas y desafios del desarrollo sostenible. Santiago de Chile: CEPAL.

Convenio Andrés Bello (CAF) (2014). Índice de vulnerabilidad y adaptación al cambio climático en la región de América Latina y el Caribe. Caracas: CAF.

Farinós Dasi, J. (2008). Gobernanza territorial para el desarrollo sostenible: estado de la cuestión y agenda. Boletin de la AGE, (46), 11-32.

Farinós Dasi, J. (2015). Desarrollo territorial y gobernanza: refinando significados desde el debate teórico pensando en la práctica. Un intento de aproximación fronética. DRd-Desenvolvimento Regional em debate, 5(2), 4-24.

Freigedo, M. y Milanesi, A. (2016). Visión 2050: Tacuarembó en la Región Norte: reporte de diagnóstico temático en el ámbito de Gobernanza e Institucionalidad para el departamento de Tacuarembó y la región Norte. Montevideo: OpP.

Germanwatch (2019). Índice de Riesgo Climático Global 2019. Bonn: Brot für die Welt. Recuperado de https://www.germanwatch.org/sites/germanwatch.org/files/Indice\%20de\%20Riesgo\%20
Climatico\%20Global\%202019\%20-\%20Resumen_o.pdf

Instituto Nacional de Investigaciones Agropecuarias (INIA) (2018). Unidad GRAS del INIA: 20 años de trayectoria. Revista INIA, (Junio), 63-68.

Kooiman, J. (2003). Governing as Governance. London: Sage.

Kronsell, A., Khan, J. y Hildingsson R. (2019). Actor relations in climate policymaking: Governing decarbnisation in a corporatist green state. Environmental Policy and Governance. 10.1002/eet.1867.

Laurnaga, M. E. y Pastori, N. (2016). Informe de Consultoría. Ámbito Sociodemográfico Salud, Educación, Cultura y Trabajo para "Visión 2050: Tacuarembó en la Región Norte.

Lipset, S. M. y Rokkan, S. (1967). Cleavage structures, party systems and voter alignments: An introduction. En S. M. Lipset y S. Rokkan (eds.), Party systems and voter alignments (pp. 1-64). New York: Free Press.

López Salgado, M. N. (2009). Informe de consultoría. Facilitadora del Sistema Nacional de Respuesta ante el Cambio Climático. Montevideo: SNRCC.

López, N. y Larghero, S. (2019). Avance del Plan Departamental de Adaptación al Cambio Climático para Rivera y Tacuarembó. Rivera: INDRA / NATIVA / IDR /IDT / SNRCC / AECID /AUCI.

Mayntz, R. (2000). Nuevos desafíos de la teoría de Governance. Instituciones y Desarrollo, (7), 35-51.

Moncayo, E. (2002). Nuevos enfoques teóricos, evolución de las política regionales e impacto territorial de la globalización. Serie gestión pública, 27. Santiago de Chile: CEPAL.

Oficina de Planificación y Presupuesto (opp) (2017). Visión 2050: Tacuarembó en la Región Norte. Montevideo: opP.

Sistema Nacional de Respuesta al Cambio Climático (sNrcC) (2010). Plan Nacional de Respuesta al 
Cambio Climático. Diagnósticos y lineamientos estratégicos. Montevideo: SNRCC.

Sistema Nacional de Respuesta al Cambio Climático (sNRCC) (2014). Cinco años de respuestas. Ante los desafios del cambio y la variabilidad climática en el Uruguay. Montevideo: mvotma. Recuperado de https://www.cambioclimatico.gub.uy/index.php/ noticias/12-noticias/97-uruguay-no-es-marginalen-las-consecuencias-del-cambio-climatico.html

Sistema Nacional de Respuesta al Cambio Climático (sNRCC) (2017a). Política nacional de cambio climático. Montevideo: sNRCC.
Sistema Nacional de Respuesta al Cambio Climático (sNRCC) (2017b). Segundo Informe Bienal de Actualización a la Conferencia de las Partes en la Convención Marco de las Naciones Unidas sobre el Cambio Climático. Montevideo: sNRCC.

Stuhldreher, A. (2016). Desafios de la gobernanza territorial sustentable. Cambio climático y desarrollo en el Noreste del Uruguay (Documentos de Trabajo 2 del IDIIs). Montevideo: Psicolibros Waslala. 